\title{
Zwingend ohne Zwang?
}

\section{Compulsory without Compulsion?}

Autoren

Institute
Andreas Spengler ${ }^{1}$, Matthias Koller ${ }^{2}$

Psychiater und Psychotherapeut, Wunstorf

Richter am Landgericht Göttingen

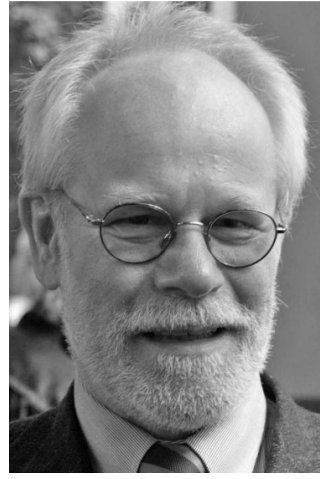

Andreas Spengler

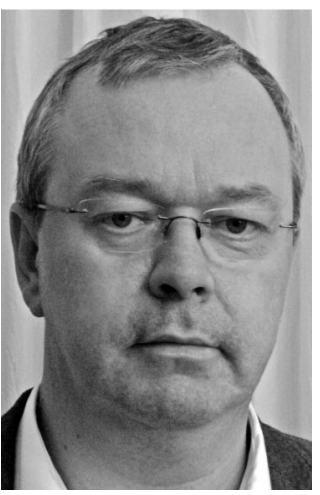

Matthias Koller

\section{Bibliografie}

DOI http://dx.doi.org/

10.1055/s-0032-1305330

Psychiat Prax 2012; 39:

313-315

(c) Georg Thieme Verlag KG

Stuttgart · New York

ISSN 0303-4259

Korrespondenzadresse

Prof. Dr. med.

Andreas Spengler

Rotdornstraße 10

31515 Wunstorf

andreas-spengler@t-online.de
Zwangsmaßnahmen in der Psychiatrie sind wieder ein Thema. In seinen Entscheidungen vom 23. März 2011 und vom 12. Oktober 2011 [1, 2] beschäftigt sich das Bundesverfassungsgericht (BVerfG) erstmals ausführlicher mit der medikamentösen Zwangsbehandlung im Maßregelvollzug (MRV), zunächst an einem Fall aus Rheinland-Pfalz. Es verwehrt dem Gesetzgeber nicht prinzipiell, solche Eingriffe zuzulassen. Aber es knüpft diese an strenge materielle und prozedurale Voraussetzungen. Der Gesetzgeber muss diese klar und bestimmt festlegen. Die zweite Entscheidung betrifft das baden-württembergische Landes-Unterbringungsgesetz, das den MRV mitregelt. Im Ergebnis entsteht der Eindruck, dass eine Zwangsmedikation praktisch kaum mehr möglich sei.

Die Entscheidungen strahlen aus und zeigen Fernwirkung: Mehrere Instanzgerichte sehen ihre Länder-Unterbringungsgesetze/PsychKG (UG/PsychKG) nicht mehr als verfassungskonform an. Und am 20. Juni 2012 hat der Bundesgerichtshof (BGH) nachgezogen und - unter Aufgabe seiner bisherigen Rechtsprechung - an die Zwangsbehandlung im Rahmen des Betreuungsrechts dieselben strengen Maßstäbe angelegt [3].

Öffentliche Reaktionen reichen von optimistischer Überinterpretation bis zur Ablehnung. Betroffenenorganisationen vertreten die absolute Unzulässigkeit der Zwangsbehandlung. Fachvertreter sehen den ärztlichen Auftrag infrage gestellt und weisen auf kaum auflösbare Zielkonflikte hin [4].

Zur Erinnerung: Spezialgesetze für Menschen mit psychischen Störungen begründen auf allen Ebenen des bürgerlichen, öffentlichen und Strafrechts einen gesellschaftlichen Doppelauftrag an die Psychiatrie: Schutz und Hilfe, aber auch Kontrolle. Wo die Grundrechte anderer verletzt werden, stößt die Selbstbestimmung an eine Grenze. Wenn eine autonome Willensbildung nicht mehr möglich ist, kann ein Eingriff geboten sein, um diese wiederherzustellen. Internationale Konventionen stehen dem nicht entgegen. Psychiatrie kommt in der Alltagsrealität nicht immer ohne Zwang aus. Sie benötigt hierzu einen klaren rechtlichen Rahmen. Individuelle Rechte stärker zu gewichten, ist ein gesamtgesellschaftlicher Trend, von dem die Psychiatrie nicht ausgenommen ist. Nun steht die Zwangsbehandlung auf dem Prüfstand. Man kann fragen, warum dies nicht früher erfolgte, nachdem die Diskussion international schon länger geführt wird und das Thema gut aufbereitet ist [5-7].

Was sagt das BVerfG? „Der schwerwiegende Eingriff in das Grundrecht aus Art.2 Abs.2 GG, der in der medizinischen Behandlung eines im Maßregelvollzug Untergebrachten gegen dessen natürlichen Willen liegt, kann auch zur Erreichung des Vollzugsziels gerechtfertigt sein " [1]. Das gilt allerdings nur, wenn der Betroffene krankheitsbedingt einsichtsunfähig ist: Eine Zwangsbehandlung - gegen seinen natürlichen Willen - ist dann zulässig

- wenn dadurch die Selbstbestimmungsfähigkeit wiederhergestellt werden soll,

- wenn sie mit dem Ziel der Selbstbestimmung und der Entlassungsfähigkeit Erfolg verspricht,

- wenn mildere Mittel nicht zur Verfügung stehen, insbesondere Aufklärungs- und Motivierungsversuche erfolglos waren und

- der Eingriff nach Art und Dauer begrenzt ist und sein Nutzen deutlich überwiegt.

- Eine Patientenverfügung darf ihr nicht entgegenstehen.

Zudem sind hohe verfahrensrechtliche Hürden zu überwinden. Neben der ärztlichen Indikationsstellung, Überwachung und Dokumentation muss nun eine unabhängige externe Prüfung stattfinden, und der Betroffene muss die Möglichkeit haben, vor Beginn der Maßnahme gerichtlichen Rechtsschutz zu suchen. Beides wird regelmäßig längere Zeit in Anspruch nehmen.

Die Wertungen des BVerfG lassen sich nicht durchweg spannungsfrei mit anderen Gesetzes- 
aufträgen und mit der Realität psychiatrischer Behandlung in Einklang bringen.

Im MRV müssen Patienten, die sich mit freiem Willen der Zwangsmedikation verweigern, eine längere Dauer der Unterbringung hinnehmen. Können sie keinen freien Willen bilden, kann eine Zwangsbehandlung zwar zulässig sein, sie darf aber nur so lange dauern, bis die Selbstbestimmungsfähigkeit wiederhergestellt ist. Danach kann ein Patient erneut - nun mit freiem Willen - ablehnen und dürfte andernfalls nur mit erklärter Einwilligung weitermediziert werden.

Diese rechtlichen Unterscheidungen gehen an der Realität der Langzeitverläufe chronischer Psychosen vorbei und verkennen die destruktive Dynamik der Krankheit für die Person selbst [8]. Das BVerfG zeigt wenig Verständnis gegenüber psychopharmakologischer Behandlung: Diese verändere seelische Abläufe und berühre in besonderem Maße den Kern der Persönlichkeit [1]. Die Behandlung gestörter psychischer Funktionen ist aber gerade ärztlicher Auftrag. Auf andere, akute (und schnell behandelbare) oder chronische psychische Störungen, etwa Manien oder Demenzen, scheint die aktuelle Rechtsprechung ohnehin nicht ohne Weiteres übertragbar zu sein.

Im MRV ist das Problem längst angekommen. Wenn auch nur in Einzelfällen, werden wieder bedrückende Verläufe und menschenunwürdige Situationen bei unbehandelten Patienten berichtet, die wegen schwerster Verhaltensprobleme nur noch dauerisoliert werden können [8]. Gewaltsituationen nehmen zu.

Die Entscheidungen des BVerfG beziehen sich aber nur auf Langzeitbehandlungen und begrenzen die Zwangsbehandlung uneingeschränkt nur „außerhalb akuter Notfälle“ [1]. Wann und wie eine medikamentöse Notfallbehandlung und Krisenintervention in Fällen akuter schwerer Selbst- oder Fremdgefährdung zulässig ist und auf welcher Rechtsgrundlage sie erfolgen soll, ist einstweilen ungeklärt.

Auch mit Zwangsmaßnahmen jenseits der Zwangsmedikation hat sich das BVerfG bisher nicht auseinandergesetzt. Fixierungen und Isolierungen können aus ärztlicher Sicht mindestens ebenso einschneidend und traumatisierend wirken. Oft sind sie keineswegs ein „milderes Mittel“. Angesichts der Gefahr fortgesetzter gravierender Fehlhandlungen - es ging um Angriffe auf Personal, um Kotschmieren und sexuelle Entgleisungen - sah das BVerfG Anfang 2012 immerhin davon ab, eine krisenintervenierende Zwangsmedikation auf dem Wege der einstweiligen Anordnung zu untersagen [9].

In der Versorgungspsychiatrie sind Sicherheit und Rechtsschutzbedürfnis, Krisenintervention und Langzeitbehandlung anders zu gewichten als im MRV. Der BGH hatte 2006 an die Zwangsbehandlung bei betreuungsrechtlicher Unterbringung strenge Anforderungen gestellt, ohne sie prinzipiell zu untersagen. Mit seinen Beschlüssen vom Juni 2012 setzt er konsequent die vom BVerfG eingeschlagene Richtung fort und verlangt eine spezifische bundesgesetzliche Grundlage [3].

Schon zuvor wurden bei betreuungsrechtlicher Unterbringung vor einer Zwangsbehandlung verlängerte Wartezeiten beobachtet. Einzelne nicht medizierte Patienten kamen in eine Dauerisolierung oder überlange Fixierung, weil sie sich manifest selbstoder fremdgefährdend verhielten, etliche verschlechterten sich klinisch. Verständigungsversuche über die Medikation bekamen aber oft eine neue Qualität: Betroffene nahmen bewusst wahr, dass ein Richter entscheidet. Nach den jüngsten BGH-Entscheidungen entsteht neue Verunsicherung. Trotz Unterbringung wurden erste Patienten wegen Unbehandelbarkeit ohne Medikation entlassen.
Viele, wenn nicht alle UG/PsychKG können nun nicht mehr als verfassungskonform gelten, weil sie die Zwangsbehandlung materiell und prozedural unzureichend regeln. Damit gerät auch die Notfallbehandlung nach sofort wirksamer Zwangseinweisung ins rechtliche Abseits. Bei vitalen Gefährdungen bleibt der rechtfertigende Notstand nach $\S 34$ StGB als einzige, aber juristisch keineswegs unbestrittene, Rechtsgrundlage. Dies schafft Rechtsunsicherheit.

Die Sicherheit von Mitpatienten scheint ein Tabuthema zu sein. Zwar sind Übergriffe äußerst selten, der „Stationsfriede“ ist aber keine autoritäre Idee, sondern Grundlage für ein therapeutisches Klima. Für Ärzte und Pflegekräfte sind Kliniken auch ein Arbeitsplatz, an dem Arbeitsschutz und -sicherheit nicht enden dürfen. Krankenstationen dürfen kein rechtsfreier Raum sein [10]. Gewerkschaftsvertreter fordern längst die Erweiterung der Gefährdungsbeurteilung nach $\S 5$ ArbSchG auf psychische Belastungen. „Burnout“ (arbeitspsychologisch definiert) wirkt sich durch Zynismus und Disengagement negativ auf die Therapie aus und kann die Griffnähe von Zwangsmaßnahmen erhöhen. Handlungsunsicherheit trägt zu Krisen bei. Weitere altbekannte Themen sind Garantenpflichten in der Suizidprävention, Aufsichtspflichten und Arzthaftung.

Legt man die Maßstäbe des BVerfG an die UG/PsychKG an, so zeigen sich Unterschiede zum MRV. Akute Erkrankungen und Gefahren gibt es zwar auch dort. Bei Patienten in der Versorgungspsychiatrie aber wäre die Alternative höchst fragwürdig, sie unbehandelt länger unterzubringen oder unnötig anderen Zwangsmitteln auszusetzen. Die UG/PsychKG gelten mit kurzer zeitlicher Reichweite, denn es geht nur um gegenwärtige, nicht anders abwendbare erhebliche Gefahren. Zwangseinweisungen nach UG/ PsychKG erfolgen meist notfallmäßig und sofort wirksam im Verwaltungsverfahren. Nicht selten kann schon am nächsten Tag freiwillig weiterbehandelt werden. Ein förmliches Unterbringungsverfahren wird dann entbehrlich. Wird doch ein Gerichtsbeschluss nötig, muss die Unterbringung möglichst kurz gehalten werden. Eine frühzeitige Medikation kann diese verkürzen und den Weg zu einer langfristigen Therapie erleichtern.

Viele Patienten erleben die Medikation unter Druck oder Zwang kurzfristig als schwere Belastung. Die Zwangsmedikation kann die Erkrankungen als sekundäre Traumatisierung besonders komplizieren, weil sie in die Körpersphäre eindringt. Etliche bräuchten für diesen Schritt mehr Zeit. Ein Abwarten kann vertrauensbildend wirken und auf längere Sicht die Annahme von Hilfe erleichtern. Ebenso wichtig ist es aber, Fixierungen zu vermeiden. Manche Betroffene fühlen sich durch körpernahe Fesselung stärker entwürdigt und belastet als durch die Medikation. Andere erleben eine Isolierung als zutiefst beängstigend. Man muss sogar fragen, ob diese Eingriffe ohne Sedierung, wenn sie unvermeidbar sind, nicht noch verletzender und auch entwürdigender werden.

Dabei ist die subjektive Wahrnehmung von Zwang dem objektiven Handlungsablauf nicht gleichzusetzen. Nur ein Teil der Patienten kann Zwangsmaßnahmen später verstehen und akzeptieren. Es darf nicht verwundern, wenn besonders vortraumatisierte Betroffene sich vehement gegen Zwang aller Art verwahren. Gerade für sie kann der Rechtsweg wichtig und heilsam sein. Es geht auch um das Gefühl von Gerechtigkeit. Es ist wichtig, wie Zwang von den Beteiligten eingesetzt wird und wieweit es gelingt, die Erfahrung therapeutisch nachzubearbeiten [7].

Literaturübersichten zur Zwangsmedikation deuten bei erheblicher Streuung auf Größenordnungen von 5\% der Aufnahmen [5]. Manche Kliniken kommen mit deutlich weniger aus. Ausgehend 
von rund 800000 psychiatrischen Fällen an 412 Kliniken im Jahr 2010 gehen viele Aufnahmen heute mit einer Zwangsmedikation einher. Die meisten davon dürften von kurzer Dauer sein. Es bleiben genügend Probleme. Die Begrenzung der personellen Ressourcen und des kostbaren Gutes Behandlungszeit führt zu einer neuen kalten Rationierung. Die vermeidbare Forensifizierung unbehandelter Psychosepatienten wird zunehmen [8]. Als Fernwirkung droht, dass mehr Schwerkranke in geschlossenen Pflegeheimen untergebracht werden, weil die Akutpsychiatrie eine effektive Behandlung nicht mehr leistet. Offen bleibt, wie die Rechtsprechung sich auf die Unterbringung in Pflegeheimen oder auch in der Somatik auswirken wird.

Eine Psychiatrie ohne Zwang ist eine uneinlösbare Vision, wenn sie nicht ihren ärztlichen und gesellschaftlichen Auftrag verraten will. Denn es wird immer Menschen geben, bei denen eine psychische Störung zur vitalen Bedrohung für sie selbst oder andere wird. Wenn Menschen ihr Recht auf Krankheit absolut ausleben, laufen sie Gefahr, aus sozialen Bezügen gänzlich herauszufallen. Einstweilen sollte die Debatte nüchtern geführt werden. Betroffene werden auch künftig ihr Recht auf Krankheit wahrnehmen und über eine Zwangsmedikation in der Klinik den Richter entscheiden lassen. Der Auftrag und die Chance, durch gute Psychiatrie Lösungen ohne Zwang zu finden, sind nicht neu. Sie erfordern zusätzliche Anstrengungen und Ressourcen.

$\mathrm{Zu}$ hoffen ist, dass die negativen Wirkungen von Zwangsmaßnahmen bewusster reflektiert werden [11-13]. Leitlinien [7] sollten im juristischen Teil aktualisiert, v.a. aber konsequent, umgesetzt werden. Zwangsmaßnahmen benötigen als Forschungsthema mehr Beachtung. Die Datenbasis muss verbessert werden, nicht nur in freiwilligen Qualitätsberichten guter Kliniken, sondern auch im Heimsektor, in der öffentlichen Gesundheitsbe- richterstattung und bei der Justiz. Die sekundäre Traumatisierung der Patienten sollte gezielt erforscht und klinisch bearbeitet werden.

Vorab ist zu fordern, dass die Gesetzgeber auf Bundes- und Landesebene ihre Verantwortung wahrnehmen und die gegenwärtige Rechtsunsicherheit beseitigen. Dazu sollte die Psychiatrie die nötigen fachlichen Grundlagen beisteuern und den nötigen politischen Druck erzeugen.

\section{Literatur}

1 BVerfG. 23. März 2011 - 2 BvR 882/09

2 BVerfG. 11. Oktober $2011-2$ BvR 633/11

3 BGH. 20. Juni 2012 - XII ZB 99/12 und XII ZB 130/12

4 Müller S, Walter H, Heinz A. Zwangsbehandlung bei psychisch Kranken: Fixieren statt behandeln? Dtsch Ärztebl 2012; 109: A198 - 202

5 Steinert T, Kallert TW. Medikamentöse Zwangsbehandlung in der Psychiatrie. Psychiat Prax 2006; 33: 160-169

6 Steinert T, BergkJ. Aggressives und gewalttätiges Verhalten. Nervenarzt 2008; 79: 359-370

7 DGPPN, Hrsg. Therapeutische Maßnahmen bei aggressivem Verhalten. Bd. 2 Behandlungsleitlinie. S2 Praxisleitlinien in Psychiatrie und Psychotherapie. Heidelberg: Steinkopff; 2010

8 Saimeh N. Zwangsbehandlung in der forensischen Psychiatrie. Soziale Psychiatrie 2012; 3: $13-17$

9 BVerfG. 10. Februar 2012 - 2 BvR 228/12

10 Böcker FM, Steinert T. Klinik als rechtsfreier Raum? Die Klinik als Ort eingeschränkten Rechtsschutzes (Kommentar). Psychiat Prax 2008; 35: $44-50$

11 Steinert T. Arbeitskreis zur Prävention von Gewalt und Zwang in der Psychiatrie. Nach 200 Jahren Psychiatrie: Sind Fixierungen in Deutschland unvermeidlich? Psychiat Prax 2011; 38: 348 - 351

12 Meise U, Frajo-Apor B. Die „subjektive Seite“ von Zwang und Gewalt in der Psychiatrie. Psychiat Prax 2011; 38: 161 - 162

13 Meise U, Frajo-Apor B, Stippler S et al. Coercion in Psychiatry - a taboo? Neuropsychiatr 2011; 25: 44-50 